Educación Física y Ciencia, vol. 19, n 1, e024, junio 2017. ISSN 2314-2561

Universidad Nacional de La Plata.

Facultad de Humanidades y Ciencias de la Educación.

Departamento de Educación Física

\title{
Incremento del dominio conceptual escolar con base en la interdisciplinariedad
}

\author{
Yamile Borrero de Castillo *; Jaime Enrique Barros Agüero ** \\ * Universidad de la Costa, Colombia; ** Universidad del Norte, Colombia | yborreroc@yahoo.es
}

\section{PALABRAS CLAVE}

Interdisciplinariedad

Herramienta metodológica

Currículo

Educación Física

Ciencias Naturales

\section{KEYWORDS}

Interdisciplinary

Methodological Tool

Curriculum

Physical Education

Natural Sciences

\begin{abstract}
RESUMEN
Este artículo es extractado de la investigación Interdisciplinariedad de la Educación Física y las Ciencias Naturales para Mejorar los Aprendizajes en Niños de Tercer Grado de Básica Primaria realizado en la CUC durante 2013 y 2014, con el objeto de dar a conocer los resultados conceptuales mejorados en el aprendizaje de las Ciencias Naturales y la Educación Física. Como herramienta metodológica, la disciplinariedad aplicada en esta investigación, tuvo su base en los aportes conceptuales de Fogarty expresados en su obra Ten Ways to Integrated Curriculum, Educational Leadership 1991. Y los Modelos disciplinarios desarrollados por Cone Theresa, Peter Werner, Stephen Cone y colaboradores para la enseñanza de la Educacíón Física. Tomaron parte del estudio 42 estudiantes, llegándose a la conclusión que la hipótesis de trabajo se cumplió, por cuanto mejoró la efectividad de la interdisciplinariedad entre la Educación Física y las Ciencias Naturales evidenciado en un mejoramiento en el dominio de los conceptos básicos de ambas disciplinas por parte de las niñas. Se concluye además que la clase interdisciplinar permite un mejor ambiente de trabajo, mucho más gratificante para el docente y alumnos dados que genera una mayor motivación que da como resultado un aprendizaje con sentido y significación. Por lo anterior, se recomienda ampliar los alcances de la investigación en Barranquilla con el ánimo de incluir un mayor número de instituciones educativas y diferentes grados que permitan dar un diagnóstico más generalizado; implementar esta investigación en otras áreas del currículo, como Ciencias Sociales, Matemática o Estética y actualizar al maestro de básica primaria, en interdisciplinariedad, de forma que se puedan implementar en la escuela este tipo de herramientas metodológicas.
\end{abstract}

\section{ABSTRACT}

This article excerpted from the Interdisciplinary Research of Physical Education and Natural Sciences to improve learning in third grade Basic Education made in Universidad de la Costa (Colombia) 2013 and 2014, in order to publicize the improved conceptual results in Learning of Natural Sciences and Physical Education. As a methodological tool, interdisciplinary applied in this research was based on the conceptual contributions of Fogarty expressed in his book Ten Ways to Integrated Curriculum. Educational Leadership Study 1991, and the Interdisciplinary Models, developed by Cone Theresa, Peter Werner, Stephen Cone, for teaching Physical Education. 42 students participated in the study which led to the conclusion that the working hypothesis was accomplished, because the effectiveness of interdisciplinary between Physical Education and Natural Sciences was improved evidenced by girls' improvement in the domain of the basic concepts of both disciplines. It can be further concluded that the interdisciplinary class allows a better working environment, more rewarding for teachers and students because it generates greater motivation that results in a learning sense and meaning. We have thought of expanding the scope of research in Barranquilla-Colombia with the aim of including a greater number of educational institutions and different levels, which would allow for a more general diagnosis. Also this research could be implemented in other areas of the curriculum such as Social Sciences, Mathematics and Aesthetics and the elementary school teacher could be updated in interdisciplinary, so that this type of methodological tools can be used in school. 


\section{Introducción}

La Educación tiene un papel importante en la comunicación de lo que es vital para la transformación de la humanidad y en la creación de una nueva cultura.

La vida es un continuo proceso de creación, de aquí que no hay un final para el proceso de aprendizaje.

El acto docente es un acto creativo y así, debe celebrarse como el momento en que profesores y estudiantes perciben el conocimiento y viven la práctica como un medio para enfrentar la vida.

La escuela es el lugar donde enseñanzas, doctrinas y opiniones convergen dando origen a una nueva cultura, con una nueva visión, basada en la diversidad de los participantes: su identidad, sus problemas, sus habilidades, sus motivaciones e intereses. La escuela orienta su función hacia la formación de hombres libres, autónomos que poseen un sentido de solidaridad, respeto, disciplina y responsabilidad, comprometidos estos con el bienestar de la sociedad de la cual forman parte.

La escuela es el lugar donde tiene lugar la transferencia del conocimiento, pero lo más importante, el lugar donde se busca el crecimiento de una mente alerta y sensible.

Finalmente la clase, entiéndase el acto pedagógico, es la forma más adecuada para el logro de los objetivos y metas de la Educación, en ella tiene lugar un proceso donde el espíritu científico y la mente creativa florecen juntos como un único movimiento que se traduce en la construcción de nuevos pensamientos, y al nacimiento de una nueva cultura.

En esta era de la "globalización”, de la "integralidad” y de la "complejidad”, la educación enfrenta diferentes desafíos y retos en la instrucción, formación y educación de las nuevas generaciones. Su naturaleza se nutre de los aportes teóricos y científicos que ofrecen las diferentes disciplinas o áreas de estudio, y en este sentido, la educación es y debe ser vista desde su naturaleza como un campo interdisciplinario.

Los complejos problemas que afronta la sociedad hoy, requieren de un enfoque interdisciplinario, para conducir la acción formativa escolar y lograr de esta manera, un aprendizaje con sentido y significación en el alumno. "Ninguna disciplina por sí sola, brinda las sabias soluciones”.

Una visión interdisciplinaria, resulta hoy, la adecuada y obligada manera de concebir la formación escolar, la cual envuelve: a). Una interdisciplinariedad curricular. (la articulación interna de las distintas disciplinas o áreas que integran el currículo. b). Una interdisciplinariedad didáctica (el empleo de distintos métodos, procesos y técnicas) y c). Una interdisciplinariedad pedagógica (la caracterización del proceso didáctico que tiene lugar en el medio donde se desarrolla el proceso enseñanza-aprendizaje. (Yves Lenoir. 2005).

La interdisciplinariedad no constituye un fin sino un medio. Su finalidad es la integración de los procesos de aprendizaje y la integración de los consiguientes saberes. La interdisciplinariedad es una exigencia interna de la ciencia. (Tamayo y Tamayo. 2011).

Desarrollar la interdisciplinariedad entre las áreas que hacen parte de un currículo educativo, aumenta la efectividad de la adquisición de aprendizajes, tanto de manera cualitativa como cuantitativa, en la medida en que se presenta de manera integrada y coherente al estudiante, los objetos de aprendizaje que por separado, se pretende que éste adquiera, desde los contenidos de cada una de las disciplinas del currículo. La anterior declaración responde a los resultados obtenidos en la investigación: Interdisciplinariedad de la Educación Física y las Ciencias Naturales para Mejorar los Aprendizajes en Niños de Tercer Grado de Educación Básica Primaria, realizada en el 2014 por José Salvador Pagano Bigio y Carlos Alberto Pérez Guardo, ambos Licenciados en Educación Física y Magisters en Ciencias de la Educación. 
Aunque en la investigación reseñada anteriormente se analizaron los matices conceptuales, socio -afectivos y psicomotores de las estudiantes de tercer grado, el presente artículo se publica con el objeto de darle a conocer a la comunidad académica y científica relacionada con la educación, los resultados concernientes con los aspectos conceptuales únicamente, puesto que los otros carices, se manifestarán en otra oportunidad.

El estudio tomó como referente los aportes conceptuales de Robin Fogarty,(1991) sobre la integración y desarrollados más tarde por Cone, T.Warner, P. y Cone, S. (1998) en especial el relacionado con el modelo de conexión, que permite realizar integraciones a través de un tema, un concepto o una destreza donde los temas de la Educación Física son los enfoques principales para las experiencias de aprendizaje y el contenido de otras ciencias es usado para aumentar, extender, ampliar o complementar el aprendizaje.

La pregunta que generó la investigación se expresó así: ¿La interdisciplinariedad de la Educación Física y las Ciencias Naturales mejoraría los aprendizajes en niños de tercer grado de básica primaria?, a lo que se planteó a manera de hipótesis de trabajo que el promedio de notas de las niñas de tercer grado del Instituto Técnico de Comercio de Barranquilla, mejoraría en un punto al desarrollar conocimientos integrados a través de unidades diseñadas interdisciplinarmente, en tiempos de tres sesiones continuas de dos horas cada una.

La anterior hipótesis fue demostrada con la aplicación de unidades de formación interdisciplinares entre las dos áreas de estudios que entraron en juego en la investigación, al aplicársele a un grupo de 41 niñas a manera de grupo contraste, mientras otro grupo de 41 niñas del mismo grado y colegio servía de control.

A través del análisis del proceso empleado en este estudio, es importante anotar, como los docentes de educación física usan estrategias didácticas para facilitar la participación activa de los estudiantes en experiencias de aprendizaje interdisciplinario. Así mismo, se establecen las formas jugadas como herramienta o medio efectivo para la adquisición, mejoramiento y comprensión de conceptos abstractos de las ciencias, como es en este caso, las Ciencias Naturales.

\section{¿Qué es la Educación Física? ¿Y Qué no es la Educación Física?}

La Educación Física es un proceso educativo enfocado al incremento y desarrollo del conocimiento humano, afectando las actividades y comportamientos de este en relación con las actividades físicas, incluyendo ejercicios, deportes, danza, actividades acuáticas y actividades al aire libre,- (Pangrazi y Darst 1.985).

Le Boulch, citado por los investigadores, manifiesta que” los ejercicios en sí mismo no son naturales o deportivos, ni son agrupables en "gimnasia estructurada" o "gimnasia funcional”, cuantitativa o cualitativa, porque el enfoque del educador y las motivaciones, necesidades e intenciones del sujeto son determinantes del desarrollo motriz “ Por su parte Naville (citado también por los investigadores) concibe la Educación Física como "una técnica reeducativa en psicopedagogía que, por el movimiento, influencia y estructura el conjunto de la personalidad del niño y corrige los trastornos psicomotores.”

La Educación Física es una experiencia curricular que envuelve al ser humano como ser único integral. Aunque todas las demás ciencias así lo pretenden y lo formulan, solo la Educación Física ofrece un mundo incalculable de oportunidades que involucran la adquisición de conocimientos, vivencias, retos, la creación artística, el desarrollo, adquisición y dominio de competencias motrices, y la solución a conflictos y desafíos. Y dado que el movimiento humano es casi ilimitado podemos decir, que las posibilidades educativas de la Educación Física son también casi ilimitadas.

La Educación Física es una materia privilegiada para contribuir a la adquisición de las competencias básicas seleccionadas e instrumentalizar el trabajo docente interdisciplinar, ya que entra su acción educativa en el desarrollo de una cultura corporal y en la propia motricidad proporcionando al alumnado experiencias que demandan interacciones con el entorno físico, social, emocional y cultural. (Mendez y colaboradores, 2009).- 
La naturaleza interna de la Educación Física, contiene los conocimientos básicos de sus siete sub-disciplinas básicas: Biomecánica, Aprendizaje Motor, Perspectiva Histórica, Fisiología del Ejercicio, Psicología Social y Estética. Basados en este conocimiento los procesos de formación e instrucción que caracterizan a la Educación Física van más allá de la mera enseñanza de movimientos y formas jugadas presentadas solas y de manera independiente, minimizadas, sin ningún valor, o solo con un valor recreacional o de diversión, como muchos pretenden conceptualizarlas. Roger Sperry decano de los neuropsicólogos norteamericanos, y citado por Howard Gardner,1993, en su estudio sobre la inteligencia cinestésica- corporal, expresa “...Uno debe pensar que la actividad mental es un medio para ejecutar acciones, en vez de que la actividad motora sea una forma subsidiaria diseñada para satisfacer las demandas de los centros superiores.”

La importancia de la Educación Física radica en el valor intrínseco de las diferentes dimensiones del ser humano estimuladas y desarrolladas a través de la naturaleza de sus contenidos, sus competencias motrices, cognitivas, afectivas y sociales.

La Educación Física es una experiencia curricular cognitiva porque fomenta y estimula la capacidad de análisis, el desarrollo del pensamiento crítico y la formulación de soluciones creativas mentales y de movimientos en la resolución de problemas y desafíos.

La Educación Física es una experiencia curricular psicológica porque ofrece al ser humano la oportunidad de enfrentar afectos, intereses, preferencias, temores, miedos y alegrías, asistiendo así al desarrollo del carácter y la personalidad.

La Educación Física es una experiencia curricular física porque a través de la práctica constante de sus conductas motrices básicas (caminar, correr, saltar...) se estimula el desarrollo, la madurez y el crecimiento físico, logrando como resultado la salud y bienestar general.

La Educación Física es una experiencia curricular orgánica porque la práctica de sus actividades de movimiento favorecen las grandes funciones vitales que sostienen la vida del hombre: Circulación, Respiración, Digestión y Reproducción.

La Educación Física es una experiencia curricular emocional porque permite al individuo establecer sus propios objetivos reales, teniendo en cuenta la individualidad. Los retos y desafíos que en ella se formulan permiten el fortalecimiento y control de emociones y quizás lo más importante sea, que a través de las actividades de movimiento el individuo no sólo compite con otros y con el medio ambiente sino consigo mismo lo que lo lleva a la reflexión y conocimiento de sí mismo.

La Educación Física es una experiencia curricular de movimiento, porque su naturaleza se basa en el movimiento. Porque su vivencia nos lleva a la conciencia de que la vida es ritmo y movimiento. Ella es en sí misma un elemento rítmico creativo. El hombre ha evolucionado y sigue evolucionando, su capacidad inherente de movimiento evoluciona con él.

Por último la Educación Física es una experiencia curricular espiritual, porque la práctica de actividades de movimiento y reposo (ejemplos el Yoga, la Meditación) permite al ser humano una trascendencia ontológica, mediante la elevación de su espíritu en búsqueda de la verdad; y una trascendencia de su corporeidad después de su tránsito, a través del legado de sus acciones y obras. La historia habla de grandes atletas; tales como: Joe Frazier, (boxeador); Shaw Johnson, (gimnasta), Nijinsky (bailarín), Martha Graham (coreógrafa)

El Desarrollo de este estudio presenta significancia por la oportunidad brindada a los investigadores y participantes con una visión de cómo el profesor de Educación Física tiene la capacidad de formular estrategias de aprendizaje presentadas de manera integral a través de la interdisciplinariedad y por la comprensión que se da en los estudiantes sobre los conceptos abstractos de las ciencias naturales, al tiempo que le permite encontrar significados válidos de la actividad física como medio efectivo para la resolución de 
las situaciones en la vida real.

\section{Reflexiones acerca de la interdisciplinariedad}

Continuemos esta reflexión definiendo a la "disciplinariedad”, como el punto de inicio de la interdisciplinariedad.

Borrero, C, A, citado por Tamayo y Tamayo, en la obra "El Proceso de la Investigación Científica” (2011) define la disciplinariedad como la "exploración realizada en un conjunto homogéneo, con el fin de producir conocimientos nuevos que, o hacen obsoletos los anteriores o los prolongan para hacerlos más completos”.

Las fuentes de contenido para un aprendizaje interdisciplinario pueden ser originadas desde el interior de la disciplina de estudio "Interdisciplinariedad Interna" o desde una fuente exterior "Interdisciplinariedad Externa”.

Desde una perspectiva interna los elementos de cada uno de las sub-disciplinas de la Educación Física, señaladas anteriormente, pueden y deben ser enseñadas a través de una interdisciplinariedad interna empoderando así al estudiante para aprender y participar de manera eficiente en experiencias de aprendizaje interdisciplinario. Dicho de otra manera los docentes diseñan experiencias interdisciplinarias internas a través de la selección de conceptos de las distintas unidades de estudio formuladas en las distintas subdisciplinas de la Educación Física. Por ejemplo: los conceptos de capacidad cardiovascular y resistencia de las sub-disciplinas base de la Educación Física, Fisiología del Ejercicio y Biomecánica, son enseñados en actividades cono la Gimnasia y deportes de Raquetas, favoreciendo así la comprensión de estos conceptos por parte del estudiante.

El aprendizaje interdisciplinario desde una interdisciplinariedad externa envuelve la enseñanza-aprendizaje del material y contenidos de la Educación Física en otras disciplinas así como también el material y contenidos de otras áreas son enseñados en los escenarios de la Educación Física. Esta conexión externa o relación entre las disciplinas significa el suficiente conocimiento del contenido de manera que el aprendizaje y aplicación de conceptos poseen un alcance que va más allá de los límites específicos de cada disciplina como resultado de un proceso de transferencia.

Las experiencias de un aprendizaje interdisciplinario, busca acabar con la rigidez de la tradicional forma de enseñar el conocimiento a través de métodos o rutas establecidas por la autonomía propia de las diferentes disciplinas de estudio.

Según Nicolescu (1999) citado por Echeverría e Hidalgo en La historia de la interdisciplinariedad en la Educación Matemática, existen tres clases de interdisciplinariedad: a) de aplicación, cuando las estrategias o técnicas de una disciplina se traspasan a otra con el objetivo de tener diferentes resultados; b) epistemológico, ocurre cuando la transferencia de los métodos de otras disciplinas impactan la epistemología de otra disciplina; c) de concepción, cuando surgen nuevas disciplinas más complejas a partir de otras más simples.

En la obra La Relación Historia-literatura en la Formación del Profesorado de Humanidades, se encuentra que "la interdisciplinariedad es un término que expresa la posibilidad de pasar los límites de una sola disciplina, permitiendo la intervención de varias escuelas de pensamiento y todo esto, debido al surgimiento de nuevos problemas que obligan a la participación y surgimiento de varias profesiones para dar soluciones”... (Suiero, Victoria, 2004) a problemas que se plantean en la adquisición de aprendizajes en las instituciones de educación. La interdisciplinariedad no es un tema común, ésta plantea la integración de saberes superando la disciplinariedad, la parcelación y fragmentación del conocimiento, producto de la división del trabajo. Corresponde a los educadores abordarla para construir estadios interdisciplinarios que 
trasciendan metodologías avanzadas.

Los anteriores conceptos, condujeron a los investigadores a cuestionar sobre la pertinencia del uso de la interdisciplinariedad, como enfoque metodológico, para la adquisición y mejoramiento en el aprendizaje de los contenidos programáticos de las Ciencias Naturales y la Educación Física en estudiantes de tercer grado de educación básica primaria. En consecuencia, se propuso realizar el estudio investigativo sobre la aplicación de esta herramienta metodológica en el Instituto Técnico de Comercio de la ciudad de Barranquilla - Colombia - en el año 2014 para comprobar su efectividad.

En el marco de ideas para el diseño de un currículo orientado y enfocado en la consecución de un aprendizaje integral, se han desarrollado diferentes modelos didácticos. Nilsen (1999) citado por Cone, Werner y Cone en "Interdisciplinary Elementary Physical Education, propone que el desarrollo e implementación de un currículo basado en la concepción de que la integralidad del currículo, podría servir mejor al alumno para lograr una integración de la información que le es proveída y establecer vínculos y relaciones de temas, tópicos o áreas de estudio. Aquí también es importante señalar, la interacción humana que resulta del trabajo conjunto de profesores.

Fogarty (1991) planteó conceptos sobre la integración del curriculum y propuso 10 diferentes maneras de hacerlo, en su trabajo: “Ten ways to integrate curriculum” a lo largo de una continuidad, extensión y proyección del total proceso de enseñanza aprendizaje, desde donde se puede concluir que conceptos, contenidos, actividades, recursos además de administradores, profesores y estudiantes juegan papel importante en el diseño de un currículo integrado e interdisciplinario.

Basados en la visión de Fogarty sobre la integración y la interdisciplinariedad en la Educación se proponen tres modelos para la enseñanza de una educación integral e interdisciplinaria para la Educación Física a través del desarrollo e implementación de tres modelos: A.- El Modelo de Conexión (The Connected Model), B.- El Modelo compartido (The Shared Model) y C.- El Modelo de Asociación (The Partnership Model).Cone, T. Werner, P. y Cone, S. (1999).-

El Modelo de Conexión (The Connected Model), se caracteriza a) por la integración y conexión interna de temas y contenidos específicos del área y b) una integración en donde los conceptos, temas, y destrezas de la Educación Física son los enfoques principales para las experiencias de aprendizaje y, el contenido de otras ciencias es usado para aumentar, extender, ampliar o complementar este aprendizaje. En este modelo el profesor de Educación Física puede trabajar de manera independiente, sin el concurso de los profesores de las otras áreas. Este es el enfoque que se tomó para adelantar esta investigación.

Por su parte, en el modelo compartido (The Shared Model), el profesor de Educación Física trabaja en equipo con los profesores de otras áreas del currículo, planificando conceptos, técnicas y habilidades que forman parte del contenido curricular relacionándolos como parte de un proceso de enseñanza-aprendizaje integral y colaborativo. Este modelo ofrece simultaneidad en aulas de dos profesores o por turnos. Requiere el ajuste del plan de enseñanza para adecuarlo a los nuevos contenidos.

Por último, en el modelo de asociación o integrado (The Partnership Model) se trabaja en equipo a partir de la Educación Física y las otras disciplinas. En este modelo más complejo se requiere de una planeación más amplia que envuelve la restructuración del currículo donde los conceptos, contenidos y habilidades son mezclados o amalgamados para el logro de objetivos comunes. La asociación implica la participación activa del equipo de profesores de dos o más disciplinas. La enseñanza es simultánea incluyendo eventos macros centralizados en un tema común.

Como consecuencia del surgimiento de las competencias básicas y el aprendizaje basado en estándares ha surgido también la necesidad de exploración y análisis de las conexiones naturales y lógicas que existen 
entre los contenidos estudiados desde diferentes disciplinas para que el aprendizaje se produzca y tenga una significación en la vida del estudiante.

Basados en la Teoría de las inteligencias Múltiples, de Howard Gardner,(1983) podemos afirmar que el trabajo interdisciplinario impacta el proceso de enseñanza-aprendizaje, a través de la oportunidad que ofrece a los educadores para resolver los retos que enfrentan en el proceso didáctico para la puesta en juego de las diferentes competencias intelectuales ( o estilos de Inteligencias) que cada alumno posee ,y que lleva a la clase, estimulándolas, motivándolas y desarrollándolas. En nuestro quehacer docente encontramos entonces en una clase o grupo, alumnos que poseen una aguda sensibilidad auditiva para los sonidos, ritmos, inflexiones y métrica de las palabras. (Inteligencia Lingüística). Otros poseen la habilidad para identificar patrones rítmicos y de tono: (Inteligencia Musical). Algunos otros poseen la habilidad para reconocer y agrupar objetos, trabajar con la abstracción y seguir una cadena de razonamientos: (Inteligencia Lógicamatemática).Otro grupo poseen la habilidad para percibir con exactitud el mundo visual, para realizar transformaciones y modificaciones a las percepciones iniciales: (Inteligencia Visual-Espacial). Encontramos también alumnos que poseen la habilidad para emplear el cuerpo en formas muy diferenciadas y hábiles para propósitos expresivos y orientados a metas. Posen dominio de la fina y gruesa coordinación:( Inteligencia Kinestésica-corporal).

Finalmente, encontramos alumnos que poseen la capacidad de identificar al instante discriminaciones entre estados de ánimo, darles códigos simbólicos y utilizarlos como un medio de comprender y guiar su propia conducta: (Inteligencia Intrapersonal), mientras otros poseen la habilidad para establecer distinciones entre otros individuos y en particular en lo referente a sus estados de ánimo, temperamento, motivaciones e intenciones (Inteligencia Interpersonal).-

Desde el punto de vista educacional, Cone, Werner, Cone y Woods (1998) exponen sobre los beneficios que presenta el uso de la interdisciplinariedad como herramienta metodológica:

1.- Provee nuevas maneras de presentar y aplicar nuevos conceptos y habilidades.

2.- Estimula la habilidad para el desarrollo del pensamiento crítico como el análisis, la síntesis y la evaluación.

3.- Desarrolla en los estudiantes capacidad del trabajo colaborativo para abordar el aprendizaje.-

4.- Motiva al estudiante porque el aprendizaje es divertido y con un sentido y significación.

5.- Motiva a los profesores a la colaboración ganando así un entendimiento del contenido de otras áreas y desarrollando una relación entre colegas.

6.- Incrementa la habilidad para reconocer y aceptar múltiple perspectivas.

7.- Nutre el pensamiento divergente y creativo.

8.-Enseña al estudiante al uso y empleo de diferentes fuentes de información para abordar un tema o asunto.

9.- Permite demostrar la transferencia del aprendizaje desde un contexto a otro.

Para mayor comprensión sobre la interdisciplinariedad presentamos el concepto de Florián Dimas, (1998), quien expresa en su obra Teoría y Práctica en la Investigación y la Enseñanza, la interdisciplinariedad ante el esquema disciplinar, no es solo una novedad o la formulación de un esquema nuevo para el campo de la educación, sino en todos los campos del desarrollo humano.

El propósito ha de ser, como se esboza en este trabajo, que los contenidos curriculares estén en sintonía con una constante superación de la separación entre materias teóricas y prácticas, para el desarrollo de estructuras mentales. 
La interdisciplinariedad entre la Educación Física y las Ciencias Naturales no solo incluye la necesidad de una mejor aplicación y utilización del conocimiento en estas disciplinas, sino que se dirige a la necesidad de cambio o evolución de un sistema educativo que responda a la incidencia cada vez mayor de una realidad diversa y compleja. La interdisciplinariedad es una vía para crear, para la proactividad, para superar desafíos metodológicos y contribuir al desarrollo del ser humano.

De igual manera, Frega, manifiesta que "la interdisciplinariedad exige el logro de relaciones de reciprocidad o co-implicación entre las didácticas de cada disciplina, aceptando que el conocimiento se construye como una estructura, y la programación de la enseñanza se basa en una didáctica constructivista que le permite al sujeto cognoscente relacionar sus conocimientos. En esta relación disciplina-enseñanza-aprendizaje la didáctica es el nexo entre ambas estructuras.”

La interdisciplinariedad rompe con los límites de cada disciplina pero le da igual importancia a cada una, permitiendo una complementariedad entre ellas y facilita el proceso de aprendizaje, de manera que se entienda un problema de aprendizaje como un solo fenómeno.

La interdisciplinariedad no es la sumatoria de conocimiento, es más que eso, es la integración de conocimientos, la articulación de disciplinas para producir soluciones a los diferentes problemas de aprendizajes, acaba con las posiciones individualistas de las áreas, permite un currículo más articulado y mejor dispuesto para el conocimiento globalizado; la interdisciplinariedad permite la concertación, el entendimiento, la armonía para el beneficio de la escuela, el alumno y el medio ambiente.

Para Piaget las relaciones interdisciplinarias tienen su base en las propias relaciones epistemológicas que se establecen entre las disciplinas, que consiste en significar sus fundamentos conceptuales y sus leyes para dar soluciones a problemas que se revelan en la escuela.

A la afirmación anterior, Ángel Villarini, 1996, explica que hay que establecer la correlación disciplinaria; es decir: el currículo de cada disciplina se elabora por separado, pero, se tiene en cuenta lo que ocurre en las otras y busca correlacionar o sea, crear paralelos entre los objetivos, contenidos y actividades de los diferentes cursos.

Para la correlación disciplinaria entre la Educación Física y las Ciencias Naturales, es muy importante considerar el juego como manifestación natural de los niños que permite el desarrollo de actividades formales e informales. Los juegos tienden a construir una amplia red de dispositivos que proporciona al niño la asimilación de toda la realidad, incorporándola, reviviéndola, dominándola y compensándola.

El juego se considera la primera herramienta para el desarrollo social, para que los sujetos realicen sus primeras interacciones. Permite que el niño compare, experimente, descubra y más adelante ponga en práctica, en otras situaciones de la vida, lo aprendido en el juego. Es importante resaltar que el juego debe estar adecuado a la edad del niño, a sus necesidades y a sus posibilidades, de manera que le ayude a estructurar su personalidad.

En el juego aflora la educación cultural del niño, que está dada por todas las acciones que el niño capta de su contexto, de su medio ambiente; pero, se puede decir que además de aflorar, también se incrementa y se da un intercambio cultural con los otros niños; cada uno le aporta al crecimiento y desarrollo del otro. Sin duda alguna, el juego es una manifestación cultural que revela el comportamiento humano develando su personalidad.

El juego modifica la cultura y la cultura al juego, a medida que se va dando la relación juego-saber, éste se va interiorizando y se va convirtiendo en parte de la persona, la cultura se va enriqueciendo, en esta medida el juego se convierte en un elemento importante de socialización. 


\section{Síntesis metodológica del estudio}

Por considerarlo pertinente para el logro de los objetivos propuestos en este estudio, se seleccionó el modelo de conexión de Cone, Werner, y Cone,S para Educación Física y se fijaron los siguientes objetivos:

- Aplicar el modelo interdisciplinario de conexión, antes mencionado entre la Educación Física y las Ciencias Naturales con el propósito de verificar que permite el progreso conceptual, psicomotor y socio-afectivo de estas disciplinas en los niños de tercer grado de básica primaria, en la institución educativa, Instituto Técnico de Comercio de Barranquilla.

- Determinar el rango de superación en el dominio de los conceptos básicos de ambas disciplinas que permitan el desarrollo conceptual en los niños objeto de estudio.

Para verificar la efectividad de la herramienta metodológica aplicada, se determinó como hipótesis de trabajo que el promedio de calificaciones que obtienen los estudiantes a raíz de su rendimiento académico, en tercer grado del Instituto Técnico de Comercio de Barranquilla, mejoraría en un punto al aumentar conocimientos integrados alcanzados a través de unidades diseñadas interdisciplinarmente entre la Educación Física y las Ciencias Naturales en tiempos de tres sesiones continuas de dos horas cada una.

Las 82 estudiantes que participaron en el estudio eran de sexo femenino, con edades entre 8 y 9 años, presentaban un promedio de 3.3 en el dominio de los conceptos relacionados con las Ciencias Naturales y la Educación Física, exhibían características físicas y cognitivas consideradas normales por el departamento de psico-orientación de la institución; tampoco demostraban problemas de aprendizaje o comportamientos sociales inadecuados. Se dividieron las niñas en dos grupos de 41 cada uno, el primero considerado de control y el segundo cuasi-experimental, al que se le aplicaron las unidades integrales diseñadas.

Desde los contenidos programáticos de la Educación Física y las Ciencias Naturales establecidos por el Estado Colombiano para el tercer grado de básica primaria, se buscó la relación con base en criterios de complementariedad, semejanza y coherencia aplicados para el análisis entre investigadores y profesores de ambas disciplinas del plantel educativo; se establecieron núcleos generadores que, al final, dieron como resultado unidades de estudio interdisciplinarias; asó por ejemplo, la transferencia del conocimiento de rotación o movilidad de las articulaciones del cuerpo (manos, cadera, hombros, cintura) recae sobre el concepto científico de la rotación de la tierra reforzándolo y complementándolo.

Producto del análisis se presentan a continuación los núcleos generadores de interdisciplinariedad que dieron origen a las unidades de esta investigación.

\section{Nucleos generadores de interdisciplinariedad}

- ¿ ¿Reconoce los huesos, su constitución y su ubicación en el cuerpo humano?

- ¿Reconoce los movimientos de traslación y rotación de la tierra?

- ¿Identifica las partes del sistema respiratorio y controla la respiración en diversos estados de excitación?

- ¿ ¿Diferencia los tipos de articulación en el cuerpo humano de acuerdo con su ubicación y movilidad?

Los núcleos generadores de interdisciplinariedad se estructuraron a manera de unidades de aprendizaje con los siguientes componentes:

- Contenidos de la Educación Física

- Contenidos de las Ciencias Naturales

- Logros interdisciplinares 
- Medios didácticos

- Actividades de enseñanza aprendizaje

- Competencias interdisciplinares: psicomotoras, conceptuales y socio-afectivas.

- Evaluación: conceptual y socio afectiva.

- Tiempo de desarrollo de cada actividad.

\section{Fases del estudio}

Esta investigación se desarrolló llevando a cabo las siguientes fases:

1. Planeación de la investigación.

2. Recopilación de información.

3. Estructuración de las unidades interdisciplinarias.

4. Desarrollo y aplicación de las clases interdisciplinarias.

5. Valoración de las clases.

6. Análisis de los resultados

Esta investigación se consideró de carácter cuasi experimental en la medida que se obtuvo un grupo cuasi experimental y un grupo control para comparar más tarde los resultados. Las técnicas que se utilizaron para esta investigación fueron: conformación de los grupos de sujetos de investigación, la orientación para el aprendizaje de contenidos interdisciplinares, el control de resultados de aprendizaje con pruebas objetivas y promedio, el uso de recursos didácticos y tiempo de desarrollo de las sesiones iguales en ambos grupos, la participación de docentes de la institución educativa, previa capacitación dada por los investigadores. Los docentes orientaron las clases interdisciplinares y evaluaron a las alumnas en las tres competencias determinadas: conceptual, psicomotriz y socio afectivo. Para la evaluación de los conceptos básicos abordados en clases se aplicó una prueba de escogencia múltiple y de asociación.

Para asegurarse que esta nueva experiencia interdisciplinaria fuera relevante a los estudiantes, la profesora conductora de la clase se apoyó en el conocimiento anterior que poseían los estudiantes en ambas disciplinas en ciernes, como punto de partida para el desarrollo de las sesiones programadas.

\section{Resultados: Valoración conceptual}

Aunque se investigaron aspectos conceptuales, socio-afectivos y psicomotores, en esta oportunidad solo se presentan los resultados conceptuales encontrados y se hace en los siguientes términos:

Se consideraron cuatro aspectos en la evaluación conceptual, desde lo interdisciplinar así: a) sistema óseo más esquema corporal, b) movimientos de rotación y traslación- tiempo espacio, c) sistema respiratorio control respiratorio y d) sistema articular- ajuste postural.

El instrumento de evaluación utilizado fue la evaluación escrita de escogencia múltiple y asociación, consistente en preguntas, cuya valoración se hizo acorde a la escala institucional, por lo cual, se procesó por aspecto evaluado en un comparativo entre el grupo de control y el grupo de contraste 
Tabla 1.

Resultados evaluación conceptual grupo control vs contraste

\begin{tabular}{|l|c|c|c|c|c|}
\hline GRUPOS & $\begin{array}{c}\text { Sistema } \\
\text { Óseo }\end{array}$ & $\begin{array}{c}\text { Rotación } \\
\text { traslación }\end{array}$ & Respiración & $\begin{array}{c}\text { Sistema } \\
\text { Articular }\end{array}$ & Promedio \\
\hline $\begin{array}{l}\text { Bajo Grupo } \\
\text { Control }\end{array}$ & 0,0 & 4,9 & 2,4 & 22,0 & 7,325 \\
\hline $\begin{array}{l}\text { Bajo Grupo } \\
\text { Contraste }\end{array}$ & 51,2 & 80,5 & 36,6 & 26,8 & 48,775 \\
\hline $\begin{array}{l}\text { Básico Grupo } \\
\text { Control }\end{array}$ & 0,0 & 0,0 & 0,0 & 0,0 & 0 \\
\hline $\begin{array}{l}\text { Básico Grupo } \\
\text { Contraste }\end{array}$ & 2,4 & 0,0 & 0,0 & 0,0 & 0,6 \\
\hline $\begin{array}{l}\text { Alto Grupo } \\
\text { Control }\end{array}$ & 0,0 & 0,0 & 24,4 & 0,0 & 6,1 \\
\hline $\begin{array}{l}\text { Alto Grupo } \\
\text { Contraste }\end{array}$ & 0,0 & 0,0 & 0,0 & 0,0 & 0 \\
\hline $\begin{array}{l}\text { Superior Grupo } \\
\text { Control }\end{array}$ & 100 & 95,1 & 73,2 & 78,0 & 86,575 \\
\hline $\begin{array}{l}\text { Superior Grupo } \\
\text { Contraste }\end{array}$ & 46,3 & 19,5 & 63,4 & 73,2 & 50,6 \\
\hline $\begin{array}{l}\text { Total } \\
\text { participantes }\end{array}$ & $\mathbf{4 1}$ & $\mathbf{4 1}$ & $\mathbf{4 1}$ & $\mathbf{4 1}$ & \\
\hline
\end{tabular}

Fuente: Interdisciplinariedad de la Educación Física y las Ciencias

Nacionales para Mejorar los Aprendizajes en Niñas de Tercer Grado de Educación Básica. Pagano Bigio, José. Pérez Guardo Carlos. 2014

En la evaluación escrita, en el punto correspondiente a la ubicación de los huesos de la cabeza, los sujetos evaluados en el grupo de control, (sujetos en clase interdisciplinar de Educación Física) el 100\% se ubicó en el nivel superior, mostrando una gran asimilación de los contenidos trabajados, por otro lado el grupo de contraste, (sujetos en clase de Ciencias Naturales), el 51,2\% alcanzaron el nivel bajo, un 2,4\% en nivel básico y el 46,3\% en nivel superior. Obsérvese, que la mayoría de los sujetos pertenecientes al grupo de contraste se encontró en bajo, lo que demuestra la no consecución del aprendizaje esperado.

En la evaluación escrita, en el punto correspondiente a la ubicación de los huesos de la cabeza, el 100\% de los sujetos evaluados en el grupo control, (sujetos en clase interdisciplinar de Educación Física) se ubicó en el nivel superior, mostrando una gran asimilación de los contenidos trabajados, por otro lado el 51,2\% del grupo de contraste, (sujetos en clase de Ciencias Naturales), alcanzaron el nivel bajo, un 2,4\% un nivel básico y el 46,3\% un nivel superior; obsérvese, que la mayoría de los sujetos pertenecientes al grupo contraste, se encontró en bajo, lo que demuestra la no consecución del aprendizaje esperado. 
Figura 1. Competencia conceptual en término de calificación

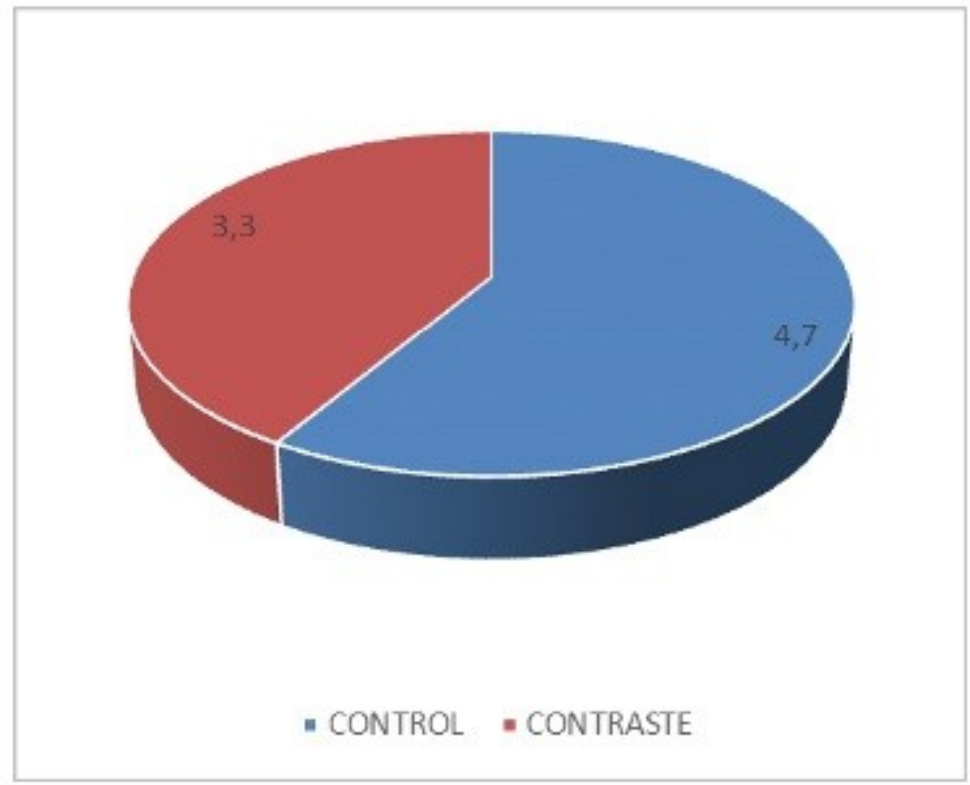

Fuente: Fuente: Interdisciplinariedad de la Educación Física y las

Ciencias Nacionales para Mejorar los Aprendizajes en Niñas de

Tercer Grado de Educación Básica. Pagano Bigio, José. Pérez Guardo

Carlos. Tamaño de la muestra 41 niñas. 2014

En este aspecto de la evaluación se verificó el reconocimiento de los movimientos de rotación y traslación encontrándose que el 4,9\% de los sujetos en el grupo control obtuvo valoración baja, mientras que el 95.1\% restante alcanzo el nivel superior. Nótese nuevamente una gran asimilación de los aprendizajes bajo el método interdisciplinar, situación que tendría justificación tal como lo explica Conde, acerca de la interdisciplinariedad cuando afirma: "Los alumnos van a adquirir conocimientos mediante tareas, debidamente programadas, diseñadas y planteadas que puedan ser bien recibidas, que asuman la intención de realizarlas y que las desarrollen con la colaboración de todos los compañeros incluido el maestro; siendo esta una forma de trabajar en equipo donde cada uno aporta un poco para alcanzar los objetivos y un aprendizaje significativo, de manera que los conocimientos adquiridos puedan ser utilizados en su vida cotidiana.”

Por otro lado, en el grupo de contraste se obtuvo por parte de los sujetos una valoración baja en el 80,5\% de los sujetos evaluados, el 19,5\% restante obtuvo valoración superior, manteniendo la tendencia mostrada en el punto anterior, en cuanto al desempeño bajo.

En el ítem de la evaluación escrita, en lo correspondiente a la respiración y el sistema respiratorio se pudo apreciar que en un 2,4\% de los estudiantes del grupo control, alcanzó un nivel de desempeño bajo, un 24,4\% alto y un 73,2\% superior, mostrando en este aspecto que algunos sujetos tuvieron alguna dificultad en el tema; sin embargo, serían muy pocos. En cuanto a los sujetos del grupo de contraste, el 36,6\% se ubicó en bajo, mientras que el 63,4\% en superior, encontrándose por debajo de los valores del grupo de control.

Estos resultados pueden obedecer como lo plantean Mertz y Aranda en su trabajo Perspectiva Interdisciplinaria en la Educación Física, "que la interdisciplinariedad entre la Educación Física y otras áreas es determinante y motivadora a la hora de integrar conocimientos, que permite una gran flexibilidad y una gran interacción entre los alumnos” situación que se evidencia en las clases desarrolladas y en los resultados de la evaluación. 
En la evaluación en el ítem correspondiente a sistema articular, interdisciplinar con ajuste postural, se puede observar que un 22\% de los sujetos en el grupo control obtuvo un desempeño bajo, el más alto de los cuatro temas abordados en las clases y evaluados de manera cognitiva, y un 78\% de los sujetos obtuvo valoración sobresaliente. El grupo contraste evidenció que un $26,8 \%$ de los sujetos presento desempeño bajo y el 73,2\% desempeño sobresaliente, obsérvese que hubo mucha similitud en los resultados de la evaluación para este contenido, factor que podría estar asociado a la motivación de los estudiantes en el tema.

\section{Conclusiones}

Después de hacer los análisis de resultados con cada una de las evaluaciones realizadas se pudo concluir que:

La hipótesis de trabajo se cumplió, por cuanto mejoró la efectividad de la interdisciplinariedad entre la Educación Física y las Ciencias Naturales evidenciado en el mejoramiento en el dominio de los conceptos básicos de ambas disciplinas por parte de los niños de tercer grado de básica primaria, que equivalió a una superación del promedio en las calificaciones del grupo contraste en 1,1 punto promedio.

El área de Educación Física se constituye en una herramienta fundamental para aplicar interdisciplinariedad con las Ciencias Naturales, por su naturaleza que facilita la motivación y el manejo de conceptos y contenidos.

Los alumnos desarrollan mejor sus estructuras conceptuales, permitiendo una mejor apropiación de los saberes.

La clase interdisciplinar permite un mejor ambiente de trabajo, mucho más gratificante para el docente y los alumnos dado que genera una mayor motivación que da como resultado un aprendizaje con mayor sentido y significación.

El modelo interdisciplinario de conexión de Cone, Werner y Cone S. aplicado entre la Educación Física y Ciencias Naturales permite el aprendizaje significativo en lo conceptual, psicomotor y socio-afectivo de estas disciplinas en los niños de tercer grado de básica primaria, en la institución educativa, Instituto Técnico de Comercio de Barranquilla.

\section{Recomendaciones}

Ampliar los alcances de la investigación en el distrito de Barranquilla o su área metropolitana con el ánimo de incluir un mayor número de instituciones educativas y diferentes grados que permitan dar un diagnóstico más generalizado.

Los docentes interdisciplinares deben tener una preparación en cuanto a una apertura mental, que rompa con los paradigmas tradicionales, con las estructuras rígidas, fraccionadas o reduccionista.

Implementar esta investigación en otras áreas del currículo escolar, tal como podría ser la Ciencias Sociales, la Matemática o la Estética.

Actualizar al maestro de básica primaria, en interdisciplinariedad, de forma que se puedan implementar en la escuela este tipo de herramientas metodológicas.

\section{Referencias}

Cabezas, M.; Meriño, F.; La interdisciplinariedad en la formación integral del maestro, Cuaderno de educación y desarrollo, Vol. 3 No 29, La Habana, (2011). 
Campo, G., Juegos pedagógicos y tradicionales, Ed. Kinesis, Armenia, (1997).

Campo, Gladys, El juego en la Educación Física básica: juegos pedagógicos y tradicionales. Editorial kinesis, Armenia (2000).

Cone, T.P.,Werner, P.,Cone, S.L., y Woods, A.M. (1998) Interdisciplinary teaching through physical Education.. Champaign.II. Human Kinetics.

Carvajal, Y., Interdisciplinariedad desafío para la educación superior y la investigación, Luna Azul, No 31, ISSN 1909-2474, Manizales, (2010).

Conde, J.; Arteaga, M.; Viciano, V., Interdisciplinariedad de las áreas en educación primaria, la Educación Física refuerzo del área de lengua castellana y literatura, Apuntes, No 51, Educación Física y deporte, Andalucía, (2011).

Conde, J. Cardenas, D. Torres, E. La concepción del profesorado sobre los factores que influyen en 1 tratamiento interdisciplinar de la Educación Física en Primaria. (2015)

Cone, T. Werner, P. Cone, S, Interdisciplinary Elemntary PHysical Eucacation.- Human Kinetics. (1998)

Cone, S. Cone, T. The Interdisciplinary puzzle. Putting the pieces all together.-Teaching Elementary Physical Education (1999) 10 (1) 8-11.-

Cortes, Ana María, La interdisciplinariedad en la educación universitaria, Anuario No 10, Buenos Aires, (2008).

Floriani, Dimas, Interdisciplinariedad: teoría y práctica en la investigación y la enseñanza, Formación Ambiental, v.10, n.23, PNUMA, México. (1998).

Fogarty, Robin, Ten ways to integrated curriculum, Educational leadership, No 49, Oregon, (1991).

Frega, Ana, Interdisciplinariedad, enfoque didáctico para la educación general, Ed. Bonun, Buenos Aires, (2007).

García, Herminia, La danza en la escuela, ed. INDEX, ISBN: 9788487339599, Barcelona, (1997).

Gardner, Howard. Estructuras de la Mente. La Teoría de las Inteligencias Múltiples, Fondo de Cultura Económica (1998)

Gessell, Arnold, El niño de 7 y 8 años, Ed. Paidós, Buenos Aires, (1978).

González, M.; Rueda, J., Investigación interdisciplinaria: urdimbres y tramas, Ed. Aula Abierta Magisterio, Bogotá, (2011).

Huizinga, Josep., Homo ludens, Ed. Fondo de cultura económica, México, (1943).

INSTECO, Proyecto educativo Institucional, (2010).

Institución educativa María Antonia Penagos, Plan de estudios del área de Ciencias Naturales, Departamento de Ciencias, Palmira,(2009).

Larroyo, Francisco, Historia general de la pedagogía, Ed. Padua, México, (1986).

Lleixá, Teresa, Desarrollo curricular para primer ciclo: Educación Física en primaria. Ed. Paidotribo, España, (1991).

Men, Marco general programas curriculares de Educación Física recreación y deportes, (1986). 
Méndez, A.; Méndez, C., Los juegos en el currículum de Educación Física: más de mil juegos para el desarrollo motor, Ed. Paidotribo, Barcelona, (2004)

Méndez, Antonio, López, Gloria, Sierra, Beatriz y Arismendieta.- Competencias Básicas: Sobre la exclusión de la competencia motriz y las aportaciones desde la Educación Física (2009).-

Meriño, F.; Cabezas, M.; La interdisciplinariedad en la formación inicial del maestro primario, Cuadernillo de educación y desarrollo, Vol. 3, No 29, La Habana, (2011).

Mertz, R.; Aranda, T., La formación docente reflexiva: perspectiva interdisciplinaria en Educación Física, Revista electrónica en investigación y docencia (REID), Brasil, (2010).

Ministerio de Educación Nacional, Lineamientos curriculares: Educación Física recreación y deporte, áreas obligatorias y fundamentales, Cooperativa editorial, Magisterio, Bogotá. (2000).

Muñoz, L.; Yovanovic, D., Programa curricular de Educación Física para preescolar, Copy graficas del Huila, Huila, (1996).

Nunes, Paulo, Educación lúdica: técnicas y juegos pedagógicos, editorial San Pablo, Brasil (1998).

Parlebas, Pierre, Problema teórico y crisis actual de la Educación Física, Revista Educación Física, Buenos aires, (1997).

Peter, Schreiner, Entrenamiento de la coordinación en el fútbol, Ed. Paidotribo, Barcelona, (2002).

Piaget, Jean, El nacimiento de la inteligencia en el niño, Ed. Aguilar, Madrid, (1969).

Piaget, Jean, Estudio de psicología genética, Ed. EMECE, Buenos Aires, (1973).

Pinzon, Marjorie, et al., Los caminos del saber: Ciencias 3, Ed. Santillana, Bogotá, (2014)

Pupo, De la Rosa, Leyva, N. La clase de Educación Física para estimular el cálculo matemático..

Ribes, Dolores, El juego infantil y su metodología. Eduforma ediciones de la U Bogotá (2011).

Romero, Gilda, Bases para la elaboración del programa de Educación Física, Ministerio de educación de la provincia de Buenos Aires, La Plata, (1963).

Salamanca, Magda, et al., Ciencias para pensar, Ed. Norma, Bogotá, (2011).

Sasano, Miguel, Cuerpo, tiempo y espacio: principios básicos de la psicomotricidad, Ed. Stadium, Buenos Aires. (2000).

Suiero, Victoria. La Relación Historia Literatura en la Formación del Profesorado de Humanidades. 2004.

Strauss, A.; Corbin, j., Bases de la investigación cualitativa, Ed. Universidad de Antioquia, Medellín, (2002).

Tamayo, Mario, Y Tamayo. El proceso de la Investigación Científica.- Quinta Edición. México. (2011).-

Vigostky, L., El desarrollo de los procesos psicológicos superiores, Ed. Critica, Barcelona, (1979).

Villarini, Ángel. El Currículo Orientado al Desarrollo Humano Integral. (1997)

Zabala, Antoni, et al., Como trabajar los contenidos procedimentales en el aula, Ed. GRAÓ de IRIF, SL, Barcelona, (2000).

Zapata, O.; Aquino. F., Psicopedagogía de la educación motriz en la etapa del aprendizaje escolar, Ed. Trilla, México, (1986). 